PROCEEDINGS OF THE

AMERICAN MATHEMATICAL SOCIETY

Volume 132, Number 5, Pages 1267-1277

S 0002-9939(03)07324-6

Article electronically published on December 12, 2003

\title{
MULTILINEAR DIFFERENTIAL OPERATORS ON MODULAR FORMS
}

\author{
MIN HO LEE \\ (Communicated by Wen-Ching Winnie Li)
}

\begin{abstract}
We construct multilinear differential operators on modular forms and prove that they are essentially unique. We also discuss certain homogeneous polynomials associated to such differential operators as well as some related multilinear differential operators that do not produce modular forms.
\end{abstract}

\section{INTRODUCTION}

Although the derivative of a modular form is not a modular form in general, certain combinations of derivatives of modular forms produce modular forms. Indeed, in [10] Rankin described the polynomials in the derivatives of modular forms for a discrete subgroup $\Gamma$ of $S L(2, \mathbb{R})$ that are again modular forms. As a special case of such polynomials, Cohen [3] studied certain bilinear operators on the graded ring of modular forms, which may be considered as noncommutative products of modular forms. These noncommutative products are known as Rankin-Cohen brackets, and they can be extended to the cases of Siegel modular forms (cf. [2], [5]) and Hilbert modular forms (see 6]). Various aspects of Rankin-Cohen brackets have been investigated recently. For example, they were studied in connection with transvectants, Heisenberg algebras, Hirota operators and other topics in mathematical physics by Olver and Sanders in [9] (see also 7], [8]). On the other hand, in 12] Unterberger discussed Rankin-Cohen brackets for nonholomorphic modular forms and their relations with quantization theory.

One of the natural ways of describing Rankin-Cohen brackets for modular forms is by way of pseudodifferential operators and formal power series called Jacobi-like forms as was discussed by Zagier in [13] (see also 4]). Indeed, there is a natural correspondence between the set of pseudodifferential operators invariant under the action of a discrete group $\Gamma \subset S L(2, \mathbb{R})$ and the set of certain sequences of modular forms for $\Gamma$, and Rankin-Cohen brackets can be constructed by using the fact that the product of two $\Gamma$-invariant pseudodifferential operators are again $\Gamma$-invariant. It is also known that $\Gamma$-invariant pseudodifferential operators also correspond to Jacobi-like forms for $\Gamma$, and the construction of Rankin-Cohen brackets can in fact be simplified if Jacobi-like forms are used. Naturally, Rankin-Cohen brackets can be extended to multilinear differential operators by considering modular forms associated to the product of more than two $\Gamma$-invariant pseudodifferential operators,

Received by the editors January 15, 2003.

2000 Mathematics Subject Classification. Primary 11F11, 11F27.

(C)2003 American Mathematical Society 
and a recent paper of Choie [1] includes the construction of such multilinear operators. One of our main goals in this paper is to show that such multilinear operators are essentially unique.

In Section 2 we construct multilinear differential operators on modular forms using Jacobi-like forms. Our construction is somewhat different from the one given in 1]. In Section 3 we prove that multilinear differential operators constructed in Section 2 are unique up to constant multiples by using theta functions with spherical coefficients. We generalize the above multilinear differential operators in Section 4 by including the ones that do not produce modular forms and study some of their properties. Finally, in Section 5 we discuss some homogeneous polynomials associated to the multilinear differential operators on modular forms constructed in Section 2,

\section{Multilinear differential operators}

Let $\Gamma$ be a discrete subgroup of $S L(2, \mathbb{R})$, and let $\mathcal{H}$ be the Poincaré upper halfplane. In this section we review Jacobi-like forms on $\mathcal{H}$ for $\Gamma$ introduced by Zagier in 13 and construct multilinear operators on the graded ring of modular forms for $\Gamma$.

The group $S L(2, \mathbb{R})$ acts on $\mathcal{H}$ by linear fractional transformations as usual. Thus, if $\gamma=\left(\begin{array}{ll}a & b \\ c & d\end{array}\right) \in S L(2, \mathbb{R})$ and $z \in \mathcal{H}$, we have

$$
\gamma z=\frac{a z+b}{c z+d}
$$

Given such $\gamma$ and $z$, an integer $k$, and a function $f: \mathcal{H} \rightarrow \mathbb{C}$, we set

$$
\left(\left.f\right|_{k} \gamma\right)(z)=f(\gamma z)(c z+d)^{-k}
$$

We now modify the usual definition of modular forms by suppressing the finiteness condition at infinity.

Definition 2.1. Given a nonnegative integer $k$, a modular form for $\Gamma$ of weight $k$ is a holomorphic function $f: \mathcal{H} \rightarrow \mathbb{C}$ satisfying

$$
\left.f\right|_{k} \gamma=f
$$

for all $\gamma \in \Gamma$. We denote by $M_{k}(\Gamma)$ the complex linear space of all modular forms for $\Gamma$ of weight $k$.

Let $\mathcal{F}$ be the ring of holomorphic functions on $\mathcal{H}$, and let $\mathcal{F}[[X]]$ be the space of formal power series in $X$ with coefficients in $\mathcal{F}$.

Definition 2.2. A Jacobi-like form for $\Gamma$ of weight $\ell \in \mathbb{Z}$ is an element $\Phi(z, X)$ of $\mathcal{F}[[X]]$ satisfying

$$
\Phi\left(\gamma z,(c z+d)^{-2} X\right)=(c z+d)^{\ell} e^{c X /(c z+d)} \Phi(z, X)
$$

for all $z \in \mathcal{H}$ and $\gamma=\left(\begin{array}{ll}a & b \\ c & d\end{array}\right) \in \Gamma$. We denote by $\mathcal{J}_{\ell}(\Gamma)$ the space of such Jacobi-like forms.

Lemma 2.3. If $f \in M_{k}(\Gamma)$, the formal power series $\widetilde{f}(z, X) \in \mathcal{F}[[X]]$ defined by

$$
\widetilde{f}(z, X)=\sum_{r=0}^{\infty} \frac{f^{(r)}(z)}{r !(r+k-1) !} X^{r}
$$

is an element of $\mathcal{J}_{k}(\Gamma)$, where $f^{(r)}$ denotes the derivative of $f$ of order $r$. 
Proof. See Section 1 in [13].

Let $\omega_{1}, \ldots, \omega_{n}$ be nonnegative integers, let $\sigma_{1}, \ldots, \sigma_{n}$ be real numbers, and set

$$
\omega=\left(\omega_{1}, \ldots, \omega_{n}\right) \in \mathbb{Z}_{+}^{n}, \quad \sigma=\left(\sigma_{1}, \ldots, \sigma_{n}\right) \in \mathbb{R}^{n},
$$

where $\mathbb{Z}_{+}$denotes the set of nonnegative integers. Throughout this paper we shall often use the multi-index notation. Thus, for example, if $\mu=\left(\mu_{1}, \ldots, \mu_{n}\right) \in \mathbb{Z}_{+}^{n}$, then we write

$$
\begin{gathered}
\sigma^{\mu}=\sigma_{1}^{\mu_{1}} \cdots \sigma_{n}^{\mu_{n}}, \quad|\sigma|=\sigma_{1}+\cdots+\sigma_{n}, \\
\mu !=\mu_{1} ! \ldots \mu_{n} !, \quad\left(\begin{array}{c}
\omega \\
\mu
\end{array}\right)=\left(\begin{array}{l}
\omega_{1} \\
\mu_{1}
\end{array}\right) \cdots\left(\begin{array}{c}
\omega_{n} \\
\mu_{n}
\end{array}\right) .
\end{gathered}
$$

In addition, if $c \in \mathbb{R}$, we shall write $c=(c, \ldots, c) \in \mathbb{R}^{n}$. Given complex-valued functions $h_{1}, \ldots, h_{n}$ on $\mathcal{H}$ and a nonnegative integer $\ell$, we set

$$
\Lambda_{\ell}^{\omega, \sigma}\left(h_{1}, \ldots, h_{n}\right)=\sum_{|\mu|=\ell} \frac{\sigma^{\mu} h^{(\mu)}}{\mu !(\mu+\omega-\mathbf{1}) !},
$$

where the summation is over $\mu=\left(\mu_{1}, \ldots, \mu_{n}\right) \in \mathbb{Z}_{+}^{n}$ with $|\mu|=\ell$ and $h^{(\mu)}=$ $h_{1}^{\left(\mu_{1}\right)} \ldots h_{n}^{\left(\mu_{n}\right)}$. Although the proof of the following proposition is essentially contained in 1, we shall include it for later reference.

Proposition 2.4. Let $f_{1}, \ldots, f_{n}$ be modular forms for $\Gamma$ with $f_{i} \in M_{\omega_{i}}(\Gamma)$ for $1 \leq i \leq n$, and assume that $|\sigma|=0$. Then $\Lambda_{\ell}^{\omega, \sigma}\left(f_{1}, \ldots, f_{n}\right)$ is a modular form for $\Gamma$ of weight $2 \ell+|\omega|$.

Proof. For each $i \in\{1, \ldots, n\}$ we denote by $\tilde{f}_{i}(z, X)$ the formal power series associated to $f_{i}$ defined as in (2.3). Then by Lemma 2.3 we see that $\widetilde{f}_{i}(z, X)$ is an element of $\mathcal{J}_{\omega_{i}}(\Gamma)$. We define the formal power series $\Phi(z, X) \in \mathcal{F}[[X]]$ by

$$
\Phi(z, X)=\prod_{i=1}^{n} \widetilde{f}_{i}\left(z, \sigma_{i} X\right) .
$$

Then by using (2.4) we see that

$$
\begin{aligned}
\Phi(z, X) & =\sum_{\mu_{1}=0}^{\infty} \cdots \sum_{\mu_{n}=0}^{\infty} \frac{\sigma_{1}^{\mu_{1}} \cdots \sigma_{n}^{\mu_{n}} f_{1}^{\left(\mu_{1}\right)}(z) \cdots f_{n}^{\left(\mu_{n}\right)}(z)}{\mu_{1} ! \cdots \mu_{n} !\left(\mu_{1}+\omega_{1}-1\right) ! \cdots\left(\mu_{n}+\omega_{n}-1\right) !} X^{\mu_{1}+\cdots+\mu_{n}} \\
& =\sum_{\ell=0}^{\infty} \sum_{|\mu|=\ell} \frac{\sigma^{\mu} f^{(\mu)}(z)}{\mu !(\mu+\omega-\mathbf{1}) !} X^{\ell} \\
& =\sum_{\ell=0}^{\infty} \Lambda_{\ell}^{\omega, \sigma}\left(f_{1}, \ldots, f_{n}\right)(z) X^{\ell}
\end{aligned}
$$

On the other hand, since $\widetilde{f}_{i}(z, X) \in \mathcal{J}_{\omega_{i}}(\Gamma)$ for each $i$, by (2.2) we obtain

$$
\begin{aligned}
\Phi\left(\gamma z,(c z+d)^{-2} X\right) & =\prod_{i=1}^{n} f_{i}\left(\gamma z,(c z+d)^{-2} \sigma_{i} X\right) \\
& =(c z+d)^{\omega_{1}+\cdots+\omega_{n}} e^{c\left(\sum_{i=1}^{n} \sigma_{i}\right) X /(c z+d)} \Phi(z, X) \\
& =(c z+d)^{|\omega|} \Phi(z, X)
\end{aligned}
$$


for all $z \in \mathcal{H}$ and $\gamma=\left(\begin{array}{ll}a & b \\ c & d\end{array}\right) \in \Gamma$. Using this and (2.5), we have

$$
\sum_{\ell=0}^{\infty} \Lambda_{\ell}^{\omega, \sigma}\left(f_{1}, \ldots, f_{n}\right)(\gamma z)(c z+d)^{-2 \ell} X^{\ell}=(c z+d)^{|\omega|} \sum_{\ell=0}^{\infty} \Lambda_{\ell}^{\omega, \sigma}\left(f_{1}, \ldots, f_{n}\right)(z) X^{\ell}
$$

Hence, by comparing the coefficients of $X^{\ell}$, we see that $\Lambda_{\ell}^{\omega, \sigma}\left(f_{1}, \ldots, f_{n}\right)$ is a modular form for $\Gamma$ of weight $2 \ell+|\omega|$, and therefore the theorem follows.

It follows from Proposition 2.4 that $\Lambda_{\ell}^{\omega, \sigma}$ determines a multilinear map from $M_{\omega_{1}}(\Gamma) \times \cdots \times M_{\omega_{n}}(\Gamma)$ to $M_{2 \ell+|\omega|}(\Gamma)$ for each $\omega=\left(\omega_{1}, \ldots, \omega_{n}\right) \in \mathbb{Z}_{+}^{n}$ and $\sigma \in \mathbb{R}^{n}$ with $|\sigma|=0$. Thus, if $M_{*}(\Gamma)=\bigoplus_{k=0}^{\infty} M_{k}(\Gamma)$ denotes the graded ring of modular forms for $\Gamma$, the map $\Lambda_{\ell}^{\omega, \sigma}$ can be regarded as a multilinear operator on $M_{*}(\Gamma)$.

\section{Theta FunCtions}

In this section we prove that the multilinear operator $\Lambda_{\ell}^{\omega, \sigma}$ in (2.4) is essentially the only multilinear differential operator of order $\ell$ by using theta functions with harmonic coefficients. The same method was used by Zagier [13] to obtain the uniqueness result for the bilinear case.

For each $j \in\{1, \ldots, n\}$, let $Q_{j}: \mathbb{Z}^{\alpha_{j}} \rightarrow \mathbb{Z}$ be a positive definite quadratic form of $\alpha_{j}$ variables, and let $P_{j}: \mathbb{Z}^{\alpha_{j}} \rightarrow \mathbb{C}$ be a spherical polynomial of even degree $\delta_{j}$ with respect to $Q_{j}$, which means that the polynomial $P_{j}$ is annihilated by the Laplacian $\triangle_{Q_{j}}$ associated to $Q_{j}$. Then it is well known that the function $\vartheta_{j}$ on $\mathcal{H}$ given by

$$
\vartheta_{j}(z)=\sum_{x \in \mathbb{Z}^{\alpha_{j}}} P_{j}(x) e^{2 \pi i z Q_{j}(x)}
$$

is a modular form of weight $\omega_{j}=\delta_{j}+\alpha_{j} / 2$ for some subgroup $\Gamma_{j} \subset S L(2, \mathbb{Z})$ of finite index. Given a positive integer $\ell$, we set

$$
\begin{aligned}
\Theta(z)=\sum_{|\rho|=\ell} C_{\rho} \vartheta^{(\rho)}(z) & =\sum_{\rho_{1}+\cdots+\rho_{n}=\ell} C_{\rho_{1}, \ldots, \rho_{n}} \vartheta_{1}^{\left(\rho_{1}\right)}(z) \cdots \vartheta_{n}^{\left(\rho_{n}\right)}(z) \\
& =\sum_{\left(x_{1}, \ldots, x_{n}\right) \in \mathbb{Z}^{\alpha_{1}+\cdots+\alpha_{n}}} \Phi\left(x_{1}, \ldots, x_{n}\right) e^{2 \pi i z \sum_{j=1}^{n} Q_{j}\left(x_{j}\right)},
\end{aligned}
$$

for all $z \in \mathcal{H}$ with $C_{\rho} \in \mathbb{C}$ for each $\rho \geq \mathbf{0}$, where $x_{j}=\left(x_{j 1}, \ldots, x_{j \alpha_{j}}\right) \in \mathbb{Z}^{\alpha_{j}}$ for $1 \leq j \leq n$ and

$$
\Phi\left(x_{1}, \ldots, x_{n}\right)=(2 \pi i)^{\ell} P_{1}\left(x_{1}\right) \cdots P_{n}\left(x_{n}\right) \sum_{\rho_{1}+\cdots+\rho_{n}=\ell} C_{\rho} Q_{1}\left(x_{1}\right)^{\rho_{1}} \cdots Q_{n}\left(x_{n}\right)^{\rho_{n}} .
$$

Then, as is well known, the function $\Theta$ on $\mathcal{H}$ is a modular form of weight $2 \ell+|\omega|$ if and only if the homogeneous polynomial $\Phi\left(x_{1}, \ldots, x_{n}\right)$ of degree $2 \ell+|\delta|$ is spherical with respect to the operator $\triangle_{Q_{1}}+\cdots+\triangle_{Q_{n}}$ (cf. [11]).

Theorem 3.1. The map $\left(f_{1}, \ldots, f_{n}\right) \mapsto \Lambda_{\ell}^{\omega, \sigma}\left(f_{1}, \ldots, f_{n}\right)$ with $|\sigma|=0$ given by (2.4) is the only n-linear differential operator of order $\ell$ from $M_{\omega_{1}}(\Gamma) \times \cdots \times M_{\omega_{n}}(\Gamma)$ to $M_{2 \ell+|\omega|}(\Gamma)$ up to a constant multiple. 
Proof. For $1 \leq j \leq n$, if $Q_{j}\left(x_{j}\right)=x_{j 1}^{2}+\cdots+x_{j \alpha_{j}}^{2}$, we have

$$
\begin{aligned}
\triangle_{Q_{j}}\left(P_{j} Q_{j}^{\rho_{j}}\right)= & \left(\triangle_{Q_{j}} P_{j}\right) Q_{j}^{\rho_{j}}+4 \rho_{j} Q_{j}^{\rho_{j}-1} \sum_{i=1}^{\alpha_{j}} x_{j 1}\left(\partial P_{j} / \partial x_{j i}\right) \\
& \quad+4 \rho_{j}\left(\rho_{j}-1\right) P_{j} Q_{j}^{\rho_{j}-1}+2 \rho_{j} \alpha_{j} P_{j} Q_{j}^{\rho_{j}-1} \\
= & 4 \rho_{j}\left(\rho_{j}-1+\delta_{j}+\alpha_{j} / 2\right) P_{j} Q_{j}^{\rho_{j}-1} \\
= & 4 \rho_{j}\left(\rho_{j}+\omega_{j}-1\right) P_{j} Q_{j}^{\rho_{j}-1} .
\end{aligned}
$$

Hence we obtain

$$
\begin{aligned}
& \left(\triangle_{Q_{1}}+\cdots+\triangle_{Q_{n}}\right) \Phi\left(x_{1}, \ldots, x_{n}\right) \\
& =4(2 \pi i)^{\ell} P_{1}\left(x_{1}\right) \cdots P_{n}\left(x_{n}\right) \sum_{|\rho|=\ell} \sum_{j=1}^{n} \rho_{j}\left(\rho_{j}+\omega_{j}-1\right) C_{\rho} \\
& \quad \times Q_{1}\left(x_{1}\right)^{\rho_{1}} \cdots Q_{n}\left(x_{n}\right)^{\rho_{n}} Q_{j}\left(x_{j}\right)^{-1}
\end{aligned}
$$

and therefore $\Theta(z)$ is a modular form if and only if

$$
\sum_{|\rho|=\ell} \sum_{j=1}^{n} \rho_{j}\left(\rho_{j}+\omega_{j}-1\right) C_{\rho} Q_{1}\left(x_{1}\right)^{\rho_{1}} \cdots Q_{n}\left(x_{n}\right)^{\rho_{n}} Q_{j}\left(x_{j}\right)^{-1}=0 .
$$

Given $\rho \in \mathbb{Z}_{+}^{n}$ with $|\rho|=\ell$ and $k \in\{1, \ldots, n\}$, we see that the coefficient of

$$
Q_{1}\left(x_{1}\right)^{\rho_{1}} \cdots Q_{n}\left(x_{n}\right)^{\rho_{n}} Q_{k}\left(x_{k}\right)^{-1}
$$

in the sum on the left-hand side of (3.1) is given by

$$
\sum_{j=1}^{n}\left(\rho_{j}+1-\delta_{j k}\right)\left(\rho_{j}+\omega_{j}-\delta_{j k}\right) C_{\rho+\boldsymbol{e}_{j}-\boldsymbol{e}_{k}}
$$

if $\rho_{k} \neq 0$, and it is zero if $\rho_{k}=0$, where $\delta_{j k}$ is the Kronecker delta and $\boldsymbol{e}_{k}$ denotes the element of $\mathbb{Z}^{n}$ with 1 in the $k$-th entry and 0 elsewhere. Let $\chi_{+}: \mathbb{R} \rightarrow \mathbb{R}$ be the characteristic function of the set of positive real numbers, and set

$$
\Xi_{k, j}=\chi_{+}\left(\rho_{j}\right)\left(\rho_{j}+1-\delta_{j k}\right)\left(\rho_{j}+\omega_{j}-\delta_{j k}\right) C_{\rho+\boldsymbol{e}_{j}-\boldsymbol{e}_{k}}
$$

for $1 \leq j \leq n$. Then $\Theta(z)$ is a modular form if and only if

$$
\sum_{j=1}^{n} \Xi_{k, j}=0
$$

for each $k \in\{1, \ldots, n\}$. For each $\rho \in \mathbb{R}_{+}^{n}$ with $|\rho|=\ell$ we set

$$
C_{\rho}=\frac{\sigma^{\rho}}{\rho !(\rho+\omega-\mathbf{1}) !}
$$


where $\sigma \in \mathbb{Z}^{n}$ with $|\sigma|=0$. If $j \neq k$ and $\rho_{j}>0$, we have

$$
\begin{aligned}
\Xi_{k, j}=\left(\rho_{j}+1\right)\left(\rho_{j}+\omega_{j}\right) \frac{\sigma_{j}^{\rho_{j}+1}}{\left(\rho_{j}+1\right) !\left(\rho_{j}+\omega_{j}\right) !} \frac{\sigma_{k}^{\rho_{k}-1}}{\left(\rho_{k}-1\right) !\left(\rho_{k}+\omega_{k}-2\right) !} \\
\quad \times \prod_{\ell \neq j, k} \frac{\sigma_{\ell}^{\rho_{\ell}}}{\rho_{\ell} !\left(\rho_{\ell}+\omega_{\ell}-1\right) !} \\
=\frac{\sigma_{j} \sigma_{k}^{\rho_{k}-1}}{\left(\rho_{k}-1\right) !\left(\rho_{k}+\omega_{k}-2\right) !} \prod_{\ell \neq k} \frac{\sigma_{\ell}^{\rho_{\ell}}}{\rho_{\ell} !\left(\rho_{\ell}+\omega_{\ell}-1\right) !} \\
=\frac{\sigma_{j} \sigma^{\rho-e_{k}}}{\left(\rho-\boldsymbol{e}_{k}\right) !\left(\rho+\omega-\boldsymbol{e}_{k}-\mathbf{1}\right) !} .
\end{aligned}
$$

On the other hand, if $j=k$ and $\rho_{j} \neq 0$, we see that

$$
\begin{aligned}
\Xi_{k, j} & =\rho_{k}\left(\rho_{k}+\omega_{k}-1\right) \prod_{\ell=1}^{n} \frac{\sigma_{\ell}^{\rho_{\ell}}}{\rho_{\ell} !\left(\rho_{\ell}+\omega_{\ell}-1\right) !} \\
& =\frac{\sigma_{k} \sigma^{\rho-e_{k}}}{\left(\rho-\boldsymbol{e}_{k}\right) !\left(\rho+\omega-\boldsymbol{e}_{k}-\mathbf{1}\right) !} .
\end{aligned}
$$

Hence the left-hand side of (3.2) becomes

$$
\sum_{j=1}^{n} \Xi_{k, j}=\frac{\sigma^{\rho-e_{k}}\left(\sigma_{1}+\cdots+\sigma_{n}\right)}{\left(\rho-e_{k}\right) !\left(\rho+\omega-e_{k}-\mathbf{1}\right) !} .
$$

Since $|\sigma|=\sigma_{1}+\cdots+\sigma_{n}=0$, it follows that $C_{\rho}$ given by (3.3) satisfies (3.2). In order to prove uniqueness up to constant multiples we assume that $C_{\rho}$ satisfies (3.2). If $\rho_{1} \neq 0$, by applying (3.2) for $k=1$ we can express $C_{\rho}$ as a linear combination of coefficients of the form $C_{\mu}$ with $\mu=\left(\mu_{1}, \ldots, \mu_{n}\right)$ and $\mu_{1}=0$. Similarly, given such a coefficient $C_{\mu}$ with $\mu_{1}=0$, if $\mu_{2} \neq 0$, by applying (3.2) for $k=2$ we can express $C_{\mu}$ as a linear combination of coefficients of the form $C_{\nu}$ with $\nu=\left(\nu_{1}, \ldots, \nu_{n}\right)$ and $\nu_{1}=\nu_{2}=0$. By induction we see that each coefficient $C_{\rho}$ can be written as a constant multiple of $C_{\ell \boldsymbol{e}_{n}}$, where $\ell \boldsymbol{e}_{n}=(0, \ldots, 0, \ell) \in \mathbb{Z}_{+}^{n}$, and therefore the theorem follows.

\section{The general CASE}

Let $\Gamma$ be a discrete subgroup of $S L(2, \mathbb{R})$, and let $\omega=\left(\omega_{1}, \ldots, \omega_{n}\right) \in \mathbb{Z}_{+}^{n}$ and $\sigma=\left(\sigma_{1}, \ldots, \sigma_{n}\right) \in \mathbb{R}^{n}$ be as in Section 2 In the previous section, we considered the maps $\Lambda_{\ell}^{\omega, \sigma}$ given by (2.4) under the assumption that $|\sigma|=0$. In this section we discuss some properties of $\Lambda_{\ell}^{\omega, \sigma}$ when $|\sigma|$ is not necessarily zero.

Lemma 4.1. Let $k$ be an integer, and let

$$
\Phi(z, X)=\sum_{n=0}^{\infty} \phi_{n}(z) X^{n} \in \mathcal{F}[[X]]
$$

Then the formal power series $\Phi(z, X)$ is an element of $J_{k}(\Gamma)$ if and only if the coefficients $\phi_{n} \in \mathcal{F}$ satisfy

$$
\left(\left.\phi_{n}\right|_{2 n+k} \gamma\right)(z)=\sum_{r=0}^{n} \frac{1}{r !}\left(\frac{c}{c z+d}\right)^{r} \phi_{n-r}(z)
$$

for all $n \geq 0, z \in \mathcal{H}$, and $\gamma=\left(\begin{array}{ll}a & b \\ c & d\end{array}\right) \in \Gamma$. 
Proof. This follows from Equation (15) in [13].

Let $f_{1}, \ldots, f_{n}$ be modular forms for $\Gamma$ with $f_{i} \in M_{\omega_{i}}(\Gamma)$ for each $i$. If $|\sigma|=$ $\sum_{i=1}^{n} \sigma_{i}=0$ and if $\Lambda_{\ell}^{\omega, \sigma}$ is as in (2.4), by Proposition 2.4 we see that for each $\ell$ the function

$$
\Lambda_{\ell}^{\omega, \sigma}(f)=\Lambda_{\ell}^{\omega, \sigma}\left(f_{1}, \ldots, f_{n}\right)
$$

satisfies the transformation formula

$$
\left.\Lambda_{\ell}^{\omega, \sigma}(f)\right|_{2 \ell+|\omega|} \gamma=\Lambda_{\ell}^{\omega, \sigma}(f)
$$

for all $\gamma \in \Gamma$, where we used the notation in (2.1). Although the functions $\Lambda_{\ell}^{\omega, \sigma}(f)$ are not modular forms in general for an arbitrary $\sigma \in \mathbb{R}^{n}$, the next theorem shows that they still satisfy a certain transformation formula with respect to the action of $\Gamma$ on $\mathcal{H}$ which generalizes 4.1).

Theorem 4.2. Let $f_{1}, \ldots, f_{n}$ be modular forms for $\Gamma$ with $f_{i} \in M_{\omega_{i}}(\Gamma)$ for each $i$, and let $\sigma \in \mathbb{R}^{n}$. Then the functions $\Lambda_{\ell}^{\omega, \sigma}(f)=\Lambda_{\ell}^{\omega, \sigma}\left(f_{1}, \ldots, f_{n}\right): \mathcal{H} \rightarrow \mathbb{C}$ satisfy the relation

$$
\left(\left.\Lambda_{\ell}^{\omega, \sigma}(f)\right|_{2 \ell+|\omega|} \gamma\right)(z)=\sum_{r=0}^{\ell} \frac{1}{r !}\left(\frac{|\sigma| c}{c z+d}\right)^{r} \Lambda_{\ell-r}^{\omega, \sigma}(f)(z)
$$

for all $z \in \mathcal{H}, \gamma=\left(\begin{array}{ll}a & b \\ c & d\end{array}\right) \in \Gamma$, and $\ell \geq 0$.

Proof. First, we consider the case where $|\sigma| \neq 0$. As in (2.5), if we set

$$
\Phi(z, X)=\prod_{i=1}^{n} \widetilde{f}_{i}\left(z, \sigma_{i} X\right)=\sum_{\ell=0}^{\infty} \Lambda_{\ell}^{\omega, \sigma}(f)(z) X^{\ell},
$$

then as in (2.6) we obtain

$$
\begin{aligned}
\Phi\left(\gamma z,(c z+d)^{-2} X\right) & =(c z+d)^{\omega_{1}+\cdots+\omega_{n}} e^{c|\sigma| X /(c z+d)} \Phi(z, X) \\
& =(c z+d)^{|\omega|} e^{c|\sigma| X /(c z+d)} \Phi(z, X)
\end{aligned}
$$

for all $\gamma=\left(\begin{array}{ll}a & b \\ c & d\end{array}\right) \in \Gamma$. Hence, if we set $\widetilde{\Phi}(z, X)=\Phi(z, X /|\sigma|)$, we see that the formal power series $\widetilde{\Phi}(z, X) \in \mathcal{F}[[X]]$ is an element of $\mathcal{J}_{|\omega|}(\Gamma)$. However, we have

$$
\widetilde{\Phi}(z, X)=\sum_{\ell=0}^{\infty} \Lambda_{\ell}^{\omega, \sigma}(f)(z)|\sigma|^{-\ell} X^{\ell} .
$$

Thus by Lemma 4.1 we see that $\widetilde{\Phi}(z, X) \in \mathcal{J}_{|\omega|}(\Gamma)$ if and only if

$$
\Lambda_{\ell}^{\omega, \sigma}(f)(z)|\sigma|^{-\ell}=(c z+d)^{2 \ell+|\omega|} \sum_{r=0}^{\ell} \frac{1}{r !}\left(\frac{c}{c z+d}\right)^{r} \Lambda_{\ell-r}^{\omega, \sigma}(f)(z)|\sigma|^{r-\ell}
$$

for each $\ell \geq 0$, which is equivalent to (4.2). However, we see that (4.2) makes sense even for $\sigma \in \mathbb{R}^{n}$ with $|\sigma|=0$ and that it reduces to (4.1) in this case. Hence the proof of the theorem is complete.

Now we consider pseudodifferential operators of the form $\sum_{r=0}^{\infty} \psi_{r}(z) \partial^{k-r}$ with $k \in \mathbb{Z}$ whose coefficients $\psi_{r}(z)$ are holomorphic functions on $\mathcal{H}$. Then the discrete subgroup $\Gamma \subset S L(2, \mathbb{R})$ acts on the space of such pseudodifferential operators by

$$
\gamma \cdot\left(\sum_{r=0}^{\infty} \psi_{r}(z) \partial^{k-r}\right)=\sum_{r=0}^{\infty} \psi_{r}(\gamma z)\left((c z+d)^{2} \partial\right)^{k-r}
$$


for $\gamma=\left(\begin{array}{ll}a & b \\ c & d\end{array}\right) \in \Gamma$. As was mentioned in the introduction, pseudodifferential operators that are invariant under $\Gamma$ are closely linked to modular forms for $\Gamma$ (cf. [4]). We note that

$$
\left((c z+d)^{2} \partial\right)^{\ell}=\sum_{r=0}^{\infty} r !\left(\begin{array}{c}
\ell \\
r
\end{array}\right)\left(\begin{array}{c}
\ell-1 \\
r
\end{array}\right) c^{r}(c z+d)^{2 \ell-r} \partial^{\ell-r}
$$

for each integer $\ell$ (see [4, (1.7)]).

Theorem 4.3. Let $f_{1}, \ldots, f_{n}$ be as in Theorem 4.2. and assume that $|\omega|=2 u$ for some $u \in \mathbb{Z}_{+}$. Then the pseudodifferential operator

$$
\Psi(z)=\sum_{k=u}^{\infty}(-1)^{k} k !(k-1) !|\sigma|^{u-k} \Lambda_{k-u}^{\omega, \sigma}(f)(z) \partial^{-k}
$$

is $\Gamma$-invariant.

Proof. By Theorem 4.2 it suffices to prove that the condition for $\Psi(z)$ to be $\Gamma$ invariant is equivalent to the condition (4.2). For each $\gamma=\left(\begin{array}{ll}a & b \\ c & d\end{array}\right) \in \Gamma$, we have

$$
\begin{aligned}
& \gamma^{-1} \cdot \Psi(z)= \sum_{r=u}^{\infty}(-1)^{r} r !(r-1) !|\sigma|^{u-r} \Lambda_{r-u}^{\omega, \sigma}(f)\left(\gamma^{-1} z\right)\left((-c z+u)^{2} \partial\right)^{-r} \\
&= \sum_{r=u}^{\infty}(-1)^{r} r !(r-1) !|\sigma|^{u-r} \Lambda_{r-u}^{\omega, \sigma}(f)\left(\gamma^{-1} z\right) \\
& \times \sum_{\ell=0}^{\infty} \ell !\left(\begin{array}{c}
-r \\
\ell
\end{array}\right)\left(\begin{array}{c}
-r-1 \\
\ell
\end{array}\right)(-c)^{\ell}(-c z+a)^{-2 r-\ell} \partial^{-r-\ell} \\
&= \sum_{k=u}^{\infty} \sum_{\ell=0}^{k-u}(-1)^{k-\ell}(k-\ell) !(k-\ell-1) !|\sigma|^{u-k+\ell} \Lambda_{k-\ell-u}^{\omega, \sigma}(f)\left(\gamma^{-1} z\right) \\
& \times \ell !\left(\begin{array}{c}
\ell-k \\
\ell
\end{array}\right)\left(\begin{array}{c}
\ell-k-1 \\
\ell
\end{array}\right)(-c)^{\ell}(-c z+d)^{-2 k+\ell} \partial^{-k},
\end{aligned}
$$

where we used (4.3). Hence $\Psi(z)$ is $\Gamma$-invariant if and only if

$$
\begin{aligned}
\Lambda_{k-u}^{\omega, \sigma}(f)(z)= & \sum_{\ell=0}^{k-u}(-1)^{\ell} \frac{(k-\ell) !(k-\ell-1) !}{k !(k-1) !} \ell !\left(\begin{array}{c}
\ell-k \\
\ell
\end{array}\right)\left(\begin{array}{c}
\ell-k-1 \\
\ell
\end{array}\right) \\
& \times(-c)^{\ell}(-c z+a)^{-2 k+\ell}|\sigma|^{\ell} \Lambda_{k-\ell-u}^{\omega, \sigma}(f)\left(\gamma^{-1} z\right) \\
= & \sum_{\ell=0}^{k-u} \frac{1}{\ell !} c^{\ell}(-c z+a)^{-2 k+\ell}|\sigma|^{\ell} \Lambda_{k-\ell-u}^{\omega, \sigma}(f)\left(\gamma^{-1} z\right)
\end{aligned}
$$

for all $\gamma=\left(\begin{array}{ll}a & b \\ c & d\end{array}\right) \in \Gamma$ and $\ell \geq u$; here we used the relations

$$
\left(\begin{array}{c}
\ell-k \\
\ell
\end{array}\right)=\frac{(-1)^{\ell}(k-1) !}{\ell !(k-\ell+1) !}, \quad\left(\begin{array}{c}
\ell-k-1 \\
\ell
\end{array}\right)=\frac{(-1)^{\ell} k !}{\ell !(k-\ell) !} .
$$


Hence, using $r=k-u$, we see that (4.4) is equivalent to

$$
\begin{aligned}
\left(\left.\Lambda_{r}^{\omega, \sigma}(f)\right|_{2 r+|\omega|} \gamma\right)(z)= & \sum_{\ell=0}^{k-u} \frac{1}{\ell !} c^{\ell}\left(-c\left(\frac{a z+b}{c z+d}\right)+a\right)^{-2 r-2 u+\ell} \\
& \times|\sigma|^{\ell} \Lambda_{r-u}^{\omega, \sigma}(f)(z)(c z+d)^{-2 r-|\omega|} \\
= & \sum_{\ell=0}^{r} \frac{1}{\ell !}\left(\frac{c}{c z+d}\right)^{\ell}|\sigma|^{\ell} \Lambda_{r-u}^{\omega, \sigma}(f)(z)
\end{aligned}
$$

for all $r \geq 0$, which in turn is equivalent to (4.2).

\section{Homogeneous polynomials}

In this section we construct a sequence of homogeneous polynomials associated to the multilinear operators $\Lambda_{k}^{\omega, \sigma}$ in Section 2 and discuss some of the properties of such polynomials. Such polynomials for the bilinear case were considered by Zagier in 13 .

Let $\Gamma \subset S L(2, \mathbb{R})$ and $\omega_{1}, \ldots, \omega_{n} \in \mathbb{Z}_{+}$be as in Section 2 and consider real numbers $\sigma_{1}, \ldots, \sigma_{n}$ with $\sum_{i=1}^{n} \sigma_{i}=0$. For each positive integer $k$ we denote by $P_{k}^{\omega, \sigma}(X)$ the homogeneous polynomial of degree $k$ given by

$$
\begin{aligned}
P_{k}^{\omega, \sigma}(X) & =\sum_{\mu_{1}+\cdots+\mu_{n}=k} \frac{\sigma_{1}^{\mu_{1}} \cdots \sigma_{n}^{\mu_{n}}}{\mu_{1} ! \cdots \mu_{n} !\left(\mu_{1}+\omega_{1}-1\right) ! \cdots\left(\mu_{n}+\omega_{n}-1\right) !} X_{1}^{\mu_{1}} \cdots X_{n}^{\mu_{n}} \\
& =\sum_{|\mu|=k} \frac{\sigma^{\mu}}{\mu !(\mu+\omega-1) !} X^{\mu} .
\end{aligned}
$$

Let $f_{1}, \ldots, f_{n}$ be modular forms for $\Gamma$ with $f_{i} \in M_{\omega_{i}}$ for each $i$, and let $\mathcal{H}^{n}$ be the $n$-fold product of the Poincare upper half-plane $\mathcal{H}$. Then we define the map $\widehat{f}: \mathcal{H}^{n} \rightarrow \mathcal{H}^{n}$ by

$$
\widehat{f}\left(z_{1}, \ldots, z_{n}\right)=\left(f_{1}\left(z_{1}\right), \ldots, f_{n}\left(z_{n}\right)\right)
$$

for all $\left(z_{1}, \ldots, z_{n}\right) \in \mathcal{H}^{n}$. We also denote by $\mathfrak{D}: \mathcal{H} \rightarrow \mathcal{H}^{n}$ the diagonal map given by

$$
\mathfrak{D}(z)=(z, \ldots, z) \in \mathcal{H}^{n}
$$

for $z \in \mathcal{H}$. Then for each $k \in \mathbb{Z}$ we see that the multilinear map $\Lambda_{k}^{\omega, \sigma}$ in (2.4) is given by

$$
\Lambda_{k}^{\omega, \sigma}\left(f_{1}, \ldots, f_{n}\right)=\left(P_{k}^{\omega, \sigma}\left(\partial_{z}\right) \widehat{f}\right) \circ \mathfrak{D}
$$

where $\partial_{z}=\left(\partial_{z_{1}}, \ldots, \partial_{z_{n}}\right)$.

Theorem 5.1. For each positive integer $k$ the homogeneous polynomial $P_{k}^{\omega, \sigma}(X)$ satisfies the following conditions:
(i) $P_{k}^{\omega, \sigma}(X)=\left.\frac{\left(\sum_{\ell=1}^{n} \sigma_{\ell} X_{\ell} \partial_{z \ell}^{-1}\right)^{k}\left(z^{\omega-1}\right)}{k !(\omega-\mathbf{1}) !}\right|_{z=\mathbf{1}}$;
(ii) $\left(\sum_{\ell=1}^{n} X_{\ell} \frac{\partial}{\partial X_{\ell}}\right) P_{k}^{\omega, \sigma}(X)=k P_{k}^{\omega, \sigma}(X)$;
(iii) $\sum_{\ell=1}^{n}\left(\omega_{\ell} \frac{\partial}{\partial X_{\ell}}+X_{\ell} \frac{\partial^{2}}{\partial X_{\ell}^{2}}\right) P_{k}^{\omega, \sigma}(X)=P_{k-1}^{\omega, \sigma}(X)$. 
Proof. Given a positive integer $k$, we have

$$
\begin{aligned}
& \left(\sum_{\ell=1}^{n} \sigma_{\ell} X_{\ell} \partial_{z_{\ell}}^{-1}\right)^{k}\left(z^{\omega-1}\right) \\
& \quad=\sum_{|\mu|=k} \frac{k !}{\mu_{1} ! \cdots \mu_{n} !}\left(\sigma_{1}^{\mu_{1}} X_{1}^{\mu_{1}} \partial_{z_{1}}^{-\mu_{1}}\right) \cdots\left(\sigma_{n}^{\mu_{n}} X_{n}^{\mu_{n}} \partial_{z_{n}}^{-\mu_{n}}\right)\left(z_{1}^{\omega_{1}-1} \cdots z_{n}^{\omega_{n}-1}\right) \\
& \quad=\sum_{|\mu|=k} \frac{k ! \sigma_{1}^{\mu_{1}} \cdots \sigma_{n}^{\mu_{n}} X_{1}^{\mu_{1}} \cdots X_{n}^{\mu_{n}}}{\mu_{1} ! \cdots \mu_{n} !}\left(\partial_{z_{1}}^{-\mu_{1}} z_{1}^{\omega_{1}-1}\right) \cdots\left(\partial_{z_{n}}^{-\mu_{n}} z_{n}^{\omega_{n}-1}\right) .
\end{aligned}
$$

However, for each $i \in\{1, \ldots, n\}$ we have

$$
\partial_{z_{i}}^{-\mu_{i}} z_{i}^{\omega_{i}-1}=\frac{\left(\omega_{i}-1\right) !}{\left(\omega_{i}+\mu_{i}-1\right) !} z_{i}^{\omega_{i}+\mu_{i}-1} .
$$

Hence we obtain

$$
\begin{aligned}
\left(\sum_{\ell=1}^{n} \sigma_{\ell} X_{\ell} \partial_{z_{\ell}}^{-1}\right)^{k}\left(z^{\omega-1}\right)= & \sum_{|\mu|=k} \frac{k ! \sigma_{1}^{\mu_{1}} \cdots \sigma_{n}^{\mu_{n}}\left(\omega_{1}-1\right) ! \cdots\left(\omega_{n}-1\right) !}{\mu_{1} ! \cdots \mu_{n} !\left(\mu_{1}+\omega_{1}-1\right) ! \cdots\left(\mu_{n}+\omega_{n}-1\right) !} \\
& \times z_{1}^{\omega_{1}+\mu_{1}-1} \cdots z_{n}^{\omega_{n}+\mu_{n}-1} X_{1}^{\mu_{1}} \cdots X_{n}^{\mu_{n}} \\
= & \sum_{|\mu|=k} \frac{k !(\omega-\mathbf{1}) ! \sigma^{\mu} z^{\omega+\mu-1} X^{\mu}}{\mu !(\mu+\omega-\mathbf{1}) !}
\end{aligned}
$$

and therefore (i) follows. On the other hand, we have

$$
\begin{aligned}
\left(\sum_{\ell=1}^{n}\right. & \left.X_{\ell} \frac{\partial}{\partial X_{\ell}}\right) P_{k}^{\omega, \sigma}(X) \\
& =\sum_{\ell=1}^{n} \sum_{|\mu|=k} \frac{\mu_{\ell} \sigma_{1}^{\mu_{1}} \cdots \sigma_{n}^{\mu_{n}}}{\mu_{1} ! \cdots \mu_{n} !\left(\mu_{1}+\omega_{1}-1\right) ! \cdots\left(\mu_{n}+\omega_{n}-1\right) !} X_{1}^{\mu_{1}} \cdots X_{n}^{\mu_{n}} . \\
& =\sum_{|\mu|=k}\left(\sum_{\ell=1}^{n} \mu_{\ell}\right) \frac{\sigma^{\mu} X^{\mu}}{\mu !(\mu+\omega-\mathbf{1}) !} .
\end{aligned}
$$

Thus, using this and the condition $\sum_{i=1}^{n} \mu_{i}=|\mu|=k$, we obtain (ii). As for (iii), we have

$$
\begin{aligned}
\sum_{\ell=1}^{n}\left(\omega_{\ell} \frac{\partial}{\partial X_{\ell}}+X_{\ell} \frac{\partial^{2}}{\partial X_{\ell}^{2}}\right) P_{k}^{\omega, \sigma}(X) & =\sum_{|\mu|=k} \sum_{\ell=1}^{n} \frac{\mu_{\ell}\left(\mu_{\ell}+\omega_{\ell}-1\right) \sigma^{\mu}}{\mu !(\mu+\omega-\mathbf{1}) !} X^{\mu-\boldsymbol{e}_{\ell}} \\
& =\sum_{|\mu|=k} \sum_{\ell=1}^{n} \frac{\sigma_{\ell} \sigma^{\mu-\boldsymbol{e}_{\ell}}}{\left(\mu-\boldsymbol{e}_{\ell}\right) !\left(\mu+\omega-\boldsymbol{e}_{\ell}-\mathbf{1}\right) !} X^{\mu-\boldsymbol{e}_{\ell}} \\
& =\sum_{\ell=1}^{n} \sigma_{\ell} \sum_{\left|\mu^{\prime}\right|=k-1} \frac{\sigma^{\mu^{\prime}}}{\mu^{\prime} !\left(\mu^{\prime}+\omega-\mathbf{1}\right) !} X^{\mu^{\prime}} \\
& =\left(\sum_{\ell=1}^{n} \sigma_{\ell}\right) P_{k-1}^{\omega, \sigma}(X)=P_{k-1}^{\omega, \sigma}(X),
\end{aligned}
$$

which verifies (iii). 


\section{REFERENCES}

[1] Y. Choie, Multilinear operators on Siegel modular forms of genus 1 and 2, J. Math. Anal. Appl. 232 (1999), 34-44. MR 2000a:11074

[2] Y. Choie and W. Eholzer, Rankin-Cohen operators for Jacobi and Siegel forms, J. Number Theory 68 (1998), 160-177. MR 99b:11050

[3] H. Cohen, Sums involving the values at negative integers of L-functions of quadratic characters, Math. Ann. 217 (1975), 271-285. MR 52:3080

[4] P. Cohen, Y. Manin, and D. Zagier, Automorphic pseudodifferential operators, Algebraic aspects of nonlinear systems, Birkhäuser, Boston, 1997, pp. 17-47. MR 98e:11054

[5] W. Eholzer and T. Ibukiyama, Rankin-Cohen type differential operators for Siegel modular forms, Internat. J. Math. 9 (1998), 443-463. MR 2000c:11079

[6] M. H. Lee, Hilbert modular pseudodifferential operators, Proc. Amer. Math. Soc. 129 (2001), 3151-3160. MR 2002k:11068

[7] P. Olver, Equivalence, invariants, and symmetry, Cambridge Univ. Press, Cambridge, 1995. MR 96i: 58005

[8] P. Olver, Classical invariant theory, London Mathematical Society Student Texts, No. 44, Cambridge Univ. Press, Cambridge, 1999. MR 2001g:13009

[9] P. Olver and J. Sanders, Transvectants, modular forms, and the Heisenberg algebra, Adv. in Appl. Math. 25 (2000), 252-283. MR 2001j:11016

[10] R. Rankin, The construction of automorphic forms from the derivatives of a given form, J. Indian Math. Soc. (N.S.) 20 (1956), 103-116. MR 18:571c

[11] B. Schoeneberg, Elliptic modular functions: an introduction, Die Grundlehren der mathematischen Wissenschaften, Band 203, Springer-Verlag, Heidelberg, 1974. MR 54:236

[12] A. Unterberger, Quantization and non-holomorphic modular forms, Lecture Notes in Math., vol. 1742, Springer-Verlag, Berlin, 2000. MR 2001k:11079

[13] D. Zagier, Modular forms and differential operators, Proc. Indian Acad. Sci. Math. Sci. 104 (1994), 57-75. MR 95d:11048

Department of Mathematics, University of Northern Iowa, Cedar Falls, Iowa 50614 E-mail address: lee@math.uni.edu 